\title{
The Role of Standards in Accelerating the Uptake of Artificial Intelligence in Dermatology
}

\author{
Liam J Caffery ${ }^{1,2}, \mathrm{PhD}$ \\ ${ }^{1}$ Dermatology Research Centre, The University of Queensland, Brisbane, Australia \\ ${ }^{2}$ Centre for Online Health, The University of Queensland, Brisbane, Australia
}

\section{Corresponding Author:}

Liam J Caffery, PhD

Dermatology Research Centre

The University of Queensland

37 Kent Street, Woolloongabba

Brisbane, Q4102

Australia

Phone: 61734437399

Email: 1.caffery@uq.edu.au

\begin{abstract}
Background: The use of artificial intelligence (AI) for dermatology is showing great promise in research contexts. However, the clinical use of AI in dermatology is still limited. The uptake of medical imaging standards for dermatology imaging is also limited. Standards adoption is more widespread in other imaging specialties (eg, radiology) as is the clinical use of AI. Digital Image Communication in Medicine (DICOM) is the standard for medical imaging. DICOM standardizes image formats and associated metadata. Further, DICOM facilitates interoperability between actors in the digital health ecosystem.

Objective: This study aimed to identify how medical imaging standards, in particular DICOM, can support the clinical use of AI.

Methods: Design Science Research Methodology was used to determine the role of DICOM in AI-based medical imaging workflows. Scenarios were identified and synthesized using expert consensus.

Results: The key benefits of using DICOM to improve the clinical use of AI were the potential to encode artefacts derived from the AI process as DICOM objects and store them alongside the original images. Such objects include downsized or down-sampled images, segmentation objects, or visual explainability maps (eg, class activation maps). DICOM can facilitate interoperability between actors in the medical imaging workflow pipeline and permits the inclusion of AI evidence creators in this pipeline. Owing to standardized image formats and metadata, DICOM can be beneficial for the curation of multi-institutional data sets. The key challenge of using DICOM is limited uptake in some specialties including dermatology.
\end{abstract}

Conclusions: DICOM offers potential to accelerate the clinical adoption of AI in dermatology by addressing several technological issues. More widespread uptake of DICOM in dermatology imaging is required to achieve this potential.

Conflicts of Interest: None declared.

(iproc 2022;8(1):e36890) doi: $10.2196 / 36890$

\section{KEYWORDS}

dermatology; artificial intelligence; DICOM; standards; imaging 
Edited by T Derrick; this is a non-peer-reviewed article. Submitted 28.01.22; accepted 28.01.22; published 23.02.22.

Please cite as:

Caffery LJ

The Role of Standards in Accelerating the Uptake of Artificial Intelligence in Dermatology iproc 2022;8(1):e36890

URL: https://www.iproc.org/2022/1/e36890

doi: $\underline{10.2196 / 36890}$

PMID:

CLiam J Caffery. Originally published in Iproceedings (https://www.iproc.org), 23.02.2022. This is an open-access article distributed under the terms of the Creative Commons Attribution License (https://creativecommons.org/licenses/by/4.0/), which permits unrestricted use, distribution, and reproduction in any medium, provided the original work, first published in Iproceedings, is properly cited. The complete bibliographic information, a link to the original publication on https://www.iproc.org/, as well as this copyright and license information must be included. 